\title{
Forbidden transitions in nuclear weak processes relevant to neutrino detection, nucleosynthesis and evolution of stars
}

\author{
Toshio Suzuki ${ }^{1,2, *}$, Satoshi Chiba ${ }^{3}$, Takashi Yoshida ${ }^{4}$, Koh Takahashi $^{5}$, Hideyuki Umeda ${ }^{4}$, and Ken 'ichi Nomoto $^{6}$ \\ ${ }^{1}$ Department of Physics, College of Humanities and Sciences, Nihon University, Setagaya-ku, Tokyo 156-8550, Japan \\ ${ }^{2}$ National Astronomical Observatory of Japan, Mitaka, Tokyo 181-8588, Japan \\ ${ }^{3}$ Reseach Laboratory for Nuclear Reactors, Tokyo Institute of Technology, Meguro, Tokyo 152-8550, Japan \\ ${ }^{4}$ Department of Astronomy, Graduate School of Science, The University of Tokyo, Hongo, Bunkyo-ku, Tokyo 113-0033, Japan \\ ${ }^{5}$ Max-Planck-Institut fur Gravitationsphysik (Albert-Einstein-Institute), Am Muhlenberg 1, D-14476, Potsdam-Golm, Germany \\ ${ }^{6}$ Kavli Institute for the Physics and Mathematics of the Universe (WPI), The University of Tokyo, Kashiwa, Chiba 277-8583, Japan
}

\begin{abstract}
The distribution of the spin-dipole strengths in ${ }^{16} \mathrm{O}$ and neutrino-induced reactions on ${ }^{16} \mathrm{O}$ are investigated by shell-model calculations with new shell-model Hamiltonians. Chargedcurrent and neutral-current reaction cross sections are evaluated in various particle and $y$ emission channels as well as the total ones at neutrino energies up to $E_{v} \approx 100 \mathrm{MeV}$. Effects of multiparticle emission channels, especially the ap emission channels, on nucleosynthesis of ${ }^{11} \mathrm{~B}$ and ${ }^{11} \mathrm{C}$ in core-collapse supernova explosions are investigated. The MSW neutrino oscillation effects on charged-current reaction cross sections are investigated for future supernova burst. Electron capture rates for a forbidden transition ${ }^{20} \mathrm{Ne}\left(0^{+}\right.$g.s. $) \rightarrow{ }^{20} \mathrm{~F}\left(2^{+}\right.$g.s. $)$in stellar environments are evaluated by the multipole expansion method with the use of shell model Hamiltonians, and compared with those obtained by a prescription that treats the transition as an allowed GamowTeller (GT) transition. Different electron energy dependence of the transition strengths between the two methods is found to lead to sizable differences in the weak rates of the two methods.
\end{abstract}

\section{Introduction}

Roles of Gamow-Teller (GT) transitions in nuclear weak rates at stellar environments have been investigated in various astrophysical processes. Electron-capture and $\beta$-decay rates in sd-shell nuclei have been updated, and applied to nuclear URCA processes in $\mathrm{O}-\mathrm{Ne}-\mathrm{Mg}$ core of stars with $\mathrm{M}=8-10 \mathrm{M}_{\odot}[1$ 3]. Those in fp-shell nuclei have been also updated with GXPF1J [4] and used to study synthesis of iron-group elements in Type Ia supernovae (SN) [5]. Neutrinonucleus reaction cross sections for ${ }^{12} \mathrm{C}[6,7],{ }^{13} \mathrm{C}[8]$, ${ }^{40} \mathrm{Ar}$ [9], ${ }^{56} \mathrm{Fe}$ and ${ }^{56} \mathrm{Ni}$ [10] have been updated, and applied to study $v$-process nucleosynthesis $[6,7,11]$ and $v$ properties [7,11]. In $\beta$-decays of $\mathrm{N}=126$ isotones, an important role of first-forbidden transitions on enhancing the rates compared with the FRDM model [12] was pointed out $[13,14]$, and the short half-lives were used to study r-process nucleosynthesis in corecollapse SN and binary neutron star mergers [14]. Here, we focus on forbidden transitions in ${ }^{16} \mathrm{O}$ and ${ }^{20} \mathrm{Ne}$. We discuss spin-dipole strengths in ${ }^{16} \mathrm{O}$ and $v$-induced reactions on ${ }^{16} \mathrm{O}$ in Sect. 2. In Sect. 3, e-capture rates for a second-forbidden transition in ${ }^{20} \mathrm{Ne}$ are evaluated with the multipole expansion method.

\section{$2 v$-induced reactions on ${ }^{16} \mathrm{O}$}

\subsection{Spin-dipole strength in ${ }^{16} \mathrm{O}$}

$v$-induced reactions on ${ }^{12} \mathrm{C}$ was studied with a shellmodel Hamiltonian, SFO [15], which can reproduce the
GT strength in ${ }^{12} \mathrm{C}$ and magnetic moments of $\mathrm{p}$-shell nuclei systematically. The configuration space of the SFO is $p$-sd shell, and the quenching factor for $q=$ $\mathrm{g}_{\mathrm{A}}{ }^{\text {eff }} / \mathrm{g}_{\mathrm{A}}$ is found to be close to $1, \mathrm{q}=0.95$, in contrast to the case within p-shell configurations such as the Cohen-Kurath Hamiltonian [16]. The monopole term in spin-isospin flip channel is enhanced in the SFO.

In case of ${ }^{16} \mathrm{O}$, the GT strength is small and the spindipole strength is the dominant contribution to spindependent transitions. Therefore, the p-sd cross-shell matrix elements in SFO are improved by taking into account the tensor and two-body spin-orbit components properly: the tensor and two-body spin-orbit components are replaced by those of $\pi+\rho$ mesonexchanges and $\sigma+\rho+\omega$ meson-exchanges, respectively. A new Hamiltonian thus obtained, SFO-tls [17], can reproduce low-lying energy levels of spin-dipole states in ${ }^{16} \mathrm{O}$. Calculated spin-dipole strength,

$$
\begin{aligned}
& B(S D \lambda)_{\mp}=\frac{1}{2 J_{i}+1} \sum_{f}\left|<f\left\|S_{\mp}^{\lambda}\right\| i>\right|^{2} \\
& S_{\mp \mu}^{\lambda}=r\left[Y^{1} \times \vec{\sigma}\right]_{\mu}^{\lambda} t_{\mp}
\end{aligned}
$$

in ${ }^{16} \mathrm{O}$ is shown in Fig. 1. Sum value $\mathrm{B}(\mathrm{SD} \lambda)$. is nearly proportional to $2 \lambda+1$, while the averaged energy position is the lowest (highest) for $2^{-}\left(1^{-}\right)$as explained below.

The energy-weighted sum of the strength can be formulated as the double commutator of the spin-dipole operator with the Hamiltonian as 


$$
\begin{aligned}
& E W S_{\mp}^{\lambda}=\sum_{f, \mu}\left|\left\langle f\left|S_{\mp \mu}^{\lambda}\right| i\right\rangle\right|^{2}\left(E_{f}-E_{i}\right) \\
& E W S^{\lambda}=E W S_{-}^{\lambda}+E W S_{+}^{\lambda}=\frac{1}{2}\left\{<0\left|\left[S_{-}^{\lambda \dagger},\left[H, S_{-}^{\lambda}\right]\right]\right| 0>\right. \\
& \left.+<0\left|\left[S_{+}^{\lambda \dagger},\left[H, S_{+}^{\lambda}\right]\right]\right| 0>\right\} .
\end{aligned}
$$

The $E W S^{\lambda}$ is determined by the kinetic energy, one-body spin-orbit potential and two-body spin-dependent interactions [18]. For ${ }^{16} \mathrm{O}, E W S^{\lambda}$ evaluated with the kinetic energy and one-body spin-orbit potential $V_{L S}=$ $\xi \Sigma_{i} \boldsymbol{\ell}_{i} \cdot \boldsymbol{\sigma}_{i}$ is given as

$$
E W S^{\lambda}=\frac{3}{4 \pi}(2 \lambda+1)\left(\frac{\hbar^{2}}{2 m} A+\frac{f_{\lambda}}{3} \xi<0\left|\sum_{i} r_{i}^{2}\right| 0>\right),
$$

where $f_{\lambda}=2,1$, and -1 for $\lambda=0,1$, and 2 , respectively, and ${ }^{16} \mathrm{O}$ is taken to be a LS-closed core. For ${ }^{16} \mathrm{O}$, the spin-orbit term splits them in the following order: $E W S^{2}{ }_{-} / 5<E W S_{-}^{1} / 3<E W S^{0}{ }_{-}$. The tensor interaction is attractive (repulsive) for $0^{-}$and $2^{-}\left(1^{-}\right)$[19]. The averaged energy defined by $<E_{\lambda}>=E W S^{\lambda} / B(S D \lambda)$ results in an order ; $\left.<E_{2}><<E_{0}><<E_{1}\right\rangle$.

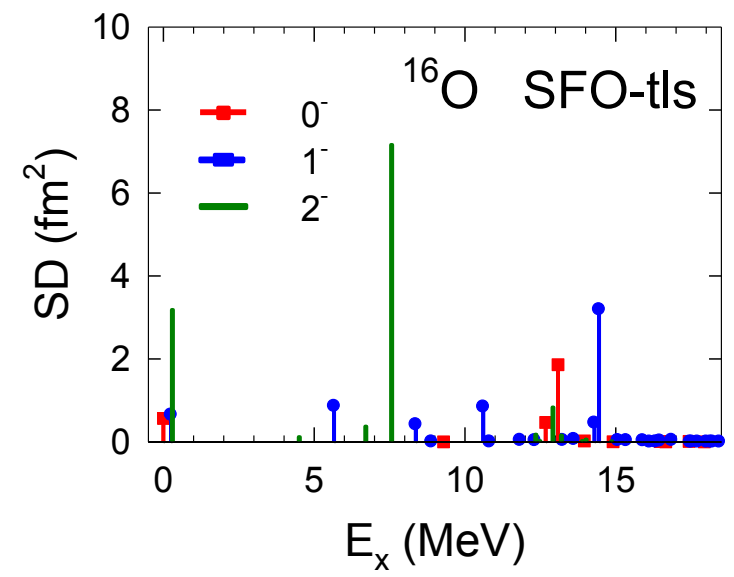

Fig. 1. Calculated spin-dipole strength in ${ }^{16} \mathrm{O}$ obtained with the SFO-tls. (Taken from Ref. [18])

\section{2. $v$-induced cross sections on ${ }^{16} \mathrm{O}$}

Charged- and neutral-current $v$-nucleus reaction cross sections on ${ }^{16} \mathrm{O}$ are evaluated by shell-model calculations with the SFO-tls [18]. The quenching for $\mathrm{g}_{\mathrm{A}}$ is taken to be $\mathrm{q}=0.95$. The total $\mu$-capture rate on ${ }^{16} \mathrm{O}$ obtained with $\mathrm{q}=0.95$ is $\lambda=11.20 \times 10^{4} \mathrm{~s}^{-1}\left(10.21 \times 10^{4} \mathrm{~s}^{-1}\right)$ for SFO-tls (SFO), which is close to the experimental value, $\lambda=11.26 \times 10^{4} \mathrm{~s}^{-1}$ [20]. Total charge-exchange cross sections for ${ }^{16} \mathrm{O}\left(v_{\mathrm{e}}, \mathrm{e}^{-}\right){ }^{16} \mathrm{~F}$ at $\mathrm{E}_{\mathrm{v}}<100 \mathrm{MeV}$ obtained for SFO-tls as well as for SFO and previous continuum-random-phase approximation (CRPA) calculation [21] are shown in Fig. 2. The multipolarities up to $\mathrm{J}=4$ are taken into account. Dominant contributions come from the transitions with $\mathrm{J}^{\pi}=2^{-}$and $1^{-}$. The cross sections for SFO-tls are enhanced compared with those of SFO, and found to be close to those of the CRPA except at $\mathrm{E}_{v}<30 \mathrm{MeV}$. Neutralcurrent cross sections for SFO-tls are also close to those of the CRPA.
Partial cross sections for various particle and $\gamma$ emission channels, including multi-particle emissions in addition to single-particle ones, are evaluated by the Hauser-Feshbach model. Cross sections for $\mathrm{p}, \mathrm{pp},{ }^{3} \mathrm{He}$, $\alpha$ and $\alpha p$ emission channels for the excitations of $2^{-}$ states are shown in Fig. 3 for SFO-tls. The proton emission channel gives the dominant contribution, while ap and aemission channels become important at higher excita-tion energies at $\mathrm{E}_{\mathrm{x}} \sim 30 \mathrm{MeV}$.

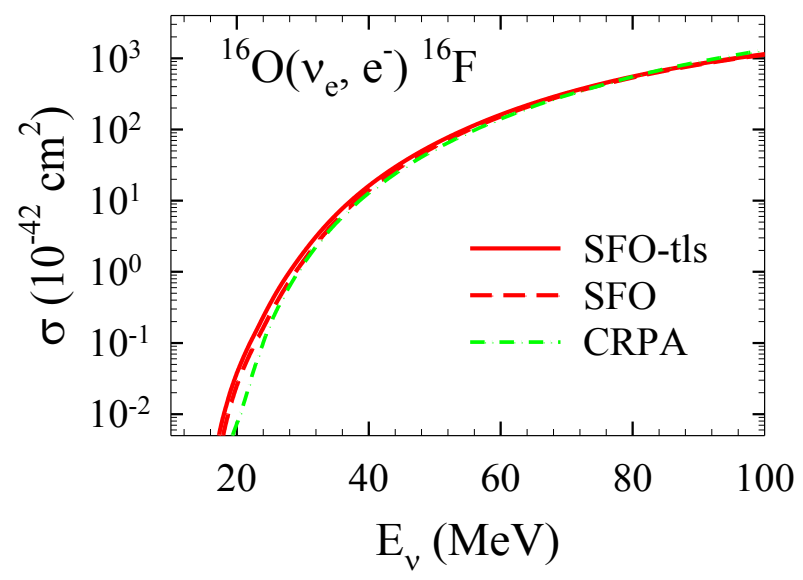

Fig. 2. Calculated total cross sections for ${ }^{16} \mathrm{O}\left(v_{\mathrm{e}}, \mathrm{e}^{-}\right){ }^{16} \mathrm{~F}$ obtained by shell-model calculations with the SFO-tls and SFO as well as CRPA calculation [21]. (Taken from Ref. [18])

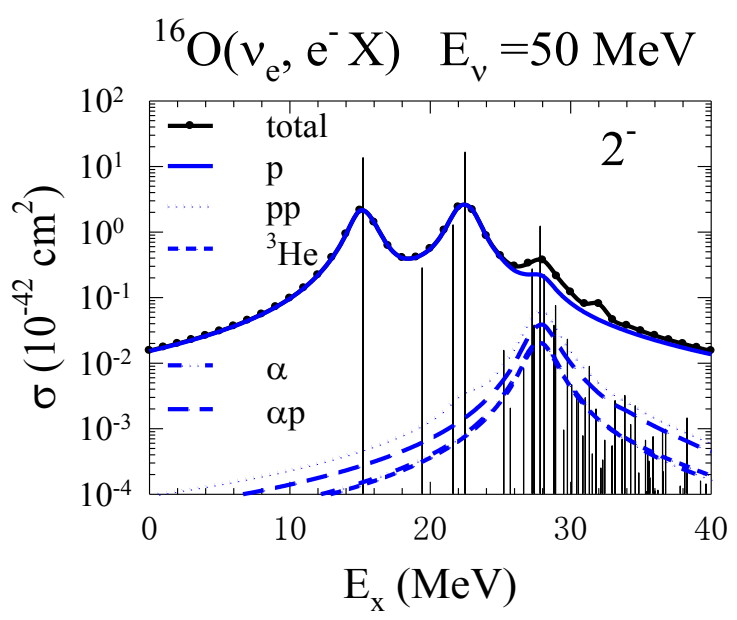

Fig. 3. Calculated partial cross sections for ${ }^{16} \mathrm{O}\left(v_{\mathrm{e}}, \mathrm{e}^{-} \mathrm{X}\right)$, with $\mathrm{X}=\mathrm{p}, \mathrm{pp},{ }^{3} \mathrm{He}, \alpha, \alpha \mathrm{p}$, via excitations of $2^{-}$states in ${ }^{16} \mathrm{~F}$ obtained by shell-model calculation with the SFO-tls. (Taken from Ref. [18])

Table 1. Production yields of ${ }^{11} \mathrm{~B}$ and ${ }^{11} \mathrm{C}$ in supernova explosion for progenitor mass of $\mathrm{M}=15 \mathrm{M}_{\odot}$. Here, "singleparticle channels" denotes $\gamma, \mathrm{p}, \mathrm{n}$ and $\alpha$ emission channels.

\begin{tabular}{|l|c|l|l|}
\hline $\begin{array}{l}\text { Production } \\
\text { yield }\left(10^{-7} \mathrm{M}_{\odot}\right)\end{array}$ & $\begin{array}{l}\text { HW92 } \\
\text { Single- } \\
\text { particle } \\
\text { channels }\end{array}$ & $\begin{array}{l}\text { SFO-tls } \\
\text { Single- } \\
\text { particle } \\
\text { channels }\end{array}$ & $\begin{array}{l}\text { SFO-tls } \\
\text { Single+Multi- } \\
\text { particle } \\
\text { channels }\end{array}$ \\
\hline${ }^{11} \mathrm{~B}$ & 2.94 & 2.92 & 3.13. \\
\hline${ }^{11} \mathrm{C}$ & 2.80 & 2.71 & 3.20 \\
\hline${ }^{11} \mathrm{~B}+{ }^{11} \mathrm{C}$ & 5.74 & 5.62 & 6.33 \\
\hline
\end{tabular}


A large branching ratio for the $\alpha p$ channel leads to the production of ${ }^{11} \mathrm{~B}$ and ${ }^{11} \mathrm{C}$ by ${ }^{16} \mathrm{O}\left(v, v^{\prime} \alpha p\right){ }^{11} \mathrm{~B}$ and ${ }^{16} \mathrm{O}\left(v_{\mathrm{e}}, \mathrm{e}^{-} \alpha \mathrm{p}\right){ }^{11} \mathrm{C}$ reactions, respectively, in addition to the ordinary reaction channels ${ }^{12} \mathrm{C}\left(v, v^{\prime} \mathrm{p}\right){ }^{11} \mathrm{~B}$ and ${ }^{12} \mathrm{C}$ $\left(v_{\mathrm{e}}, \mathrm{e}^{-} \mathrm{p}\right){ }^{11} \mathrm{C}$. The production yields of ${ }^{11} \mathrm{~B}+{ }^{11} \mathrm{C}$ in supernovae with 15 (20) $\mathrm{M}_{\odot}$ are found to be enhanced by about 13 (12)\% compared with those without the multi-particle emission channels [18] (see Table 1).

\section{$2.3 v$ oscillations and detection of supernova $v$}

The MSW matter resonance oscillations [22] occur in C- He layer of supernovae for normal (inverted) mass hierarchy in charged-current reactions induced by $v_{\mathrm{e}}\left(\bar{v}_{\mathrm{e}}\right)$. Event spectra of neutrino- ${ }^{16} \mathrm{O}$ charged-current reactions at Super-Kamiokande are evaluated for future super-nova neutrino bursts. The cross sections of the ${ }^{16} \mathrm{O}$ $\left(v_{\mathrm{e}}, \mathrm{e}^{-}\right) \mathrm{X}$ and ${ }^{16} \mathrm{O}\left(\bar{v}_{\mathrm{e}}, \mathrm{e}^{+}\right) \mathrm{X}$ reactions for each nuclear state with a different excitation energy are evaluated, and dependence of the cross sections on the mass hierarchies are examined [23]. Enhancement of expected event numbers for supernovae at $10 \mathrm{kpsc}$ away from the earth is predicted for the $v_{\mathrm{e}}\left(\bar{v}_{\mathrm{e}}\right)$-induced reaction in case of normal (inverted) hierachy [23].

\section{Electron-capture rates for a second- forbidden transition in ${ }^{20} \mathrm{Ne}$}

\subsection{Evolution of O-Ne-Mg cores in stars}

Evolutions and final fate of a star with $8-10 \mathrm{M}_{\odot}$ are sensitive to the nuclear weak rates as well as its mass. The cooling of the O-Ne-Mg core of the star produced after carbon burning occurs at certain densities by nuclear URCA processes for pairs of nuclei with $\mathrm{A}=25$ and 23 [1-3]. In later stage of the evolution, the core gets heated by $\gamma$ emissions in double e-capture processes on ${ }^{24} \mathrm{Mg}$ and ${ }^{20} \mathrm{Ne}$. It ws pointed out that a secondforbidden transition in ${ }^{20} \mathrm{Ne},{ }^{20} \mathrm{Ne}\left(0_{\text {g.s. }}{ }^{+}\right) \rightarrow{ }^{20} \mathrm{~F}\left(2_{1}{ }^{+}\right)$, becomes important at high densities $\log _{10}\left(\rho \mathrm{Y}_{\mathrm{e}}\right)=9.3-9.6$, where $Y_{e}$ is the lepton to baryon ratio, and at temperatures $\log _{10}(\mathrm{~T})<9.0$ in e-capture reactions [24]. However, the transition strength has not been accurately determined [25]. The e-capture rates for the second forbidden transition in ${ }^{20} \mathrm{Ne}$ can be important to estimate the heating of the core and to determine the final fate of stars with $8-10 \mathrm{M}_{\odot}$.

\subsection{Electron-capture rates for ${ }^{20} \mathrm{Ne}\left(0_{\text {g.s. }}{ }^{+}\right) \rightarrow{ }^{20} \mathrm{~F}$ $\left(2{ }_{1}{ }^{+}\right)$in stellar environments}

Recenty, the $\beta$-decay rate for the transition ${ }^{20} \mathrm{~F}$ $\left(2_{1}{ }^{+}\right) \rightarrow{ }^{20} \mathrm{Ne}\left(0_{\text {g.s. }}{ }^{+}\right)$was measured, and $\log f t$ value was obtained to be $10.47 \pm 0.11$ [26], which is close to the lower limt value given in NNDC [25]. The e-capture rates for the forbidden transition in ${ }^{20} \mathrm{Ne}$ were calculated assuming the transition to be an allowed GT transition [24]. Here, we evaluate the e-capture rates accurately with the multipole expansion method [27]. In this method, there are contributions from the Coulomb, longitudinal, transverse electric and axial magnetic terms with the multipolarity $\mathrm{J}^{\pi}=2^{+}$. The transition strength has a dependence on the electron energy as in the cases of first-forbidden transitions in contrast to the allowed GT transition.

Calculated e-capture rates for the forbidden transition obtained with the USDB Hamitonian are shown in Fig. 4 for the temperature $\log _{10} \mathrm{~T}=8.6$. Results obtained by the prescription assuming an allowed GT transition with the strength determined from the experimental $\log f t$ value [26] are also shown for comparison.

Sizable difference is found between the two methods [28]. The rates obtained by the GT prescription are found to be enhanced (reduced) compared with those with the USDB at $\log _{10}\left(\rho Y_{\mathrm{e}}\right)<(>)$ 9.9. This comes from the difference in the electron energy dependence of the transition strengths between the two methods. The strength for the USDB is reduced (enhanced) at $\mathrm{E}_{\mathrm{e}}<(>)$ 9.9 $\mathrm{MeV}$ compared with the GT prescription. At $\log _{10}\left(\rho Y_{e}\right)<9.8$, where the electron chemical potential is below $10 \mathrm{MeV}$, the shell-model strength is smaller than the GT one, and the rates for USDB also remain smaller than the GT one. At $\log _{10}\left(\rho Y_{e}\right) \geq 9.9$, on the other hand, the electron energy larger than $10 \mathrm{MeV}$ can contribute to the rates due to the enhancement of the chemical potential, and the rates for USDB begin to exceed the GT rate.

Effects of the Coulomb effects, that is, the screening effects on both electrons and ions [29-32], are investigated. The Coulomb effects reduce the e-capture rates and shift them toward higher density region due to an increase of the threshold energy by $\Delta Q_{C}=\mu_{C}(Z-1)$ $\mu_{C}(Z)$, where $\mu_{C}(Z)$ is the Coulomb chemical potential of the nucleus with charge number $Z$ due to the interaction of the ion with other ions in the electron background (see more details in Ref. [28]).

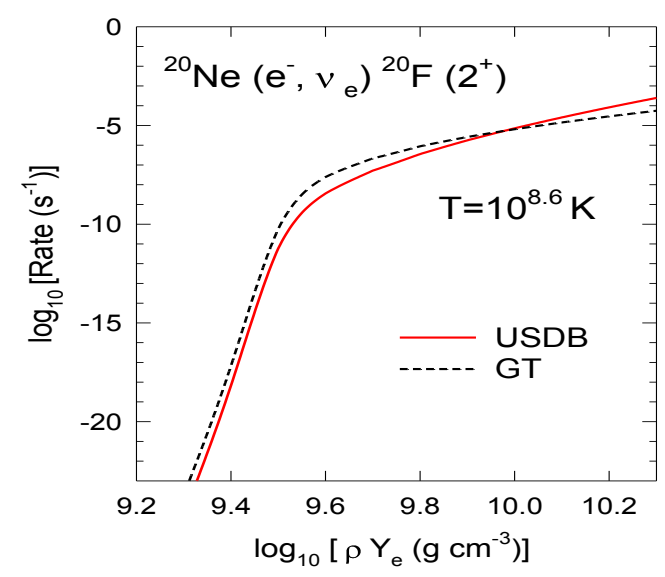

Fig. 4. Calculated e-capture rates for ${ }^{20} \mathrm{Ne}\left(\mathrm{e}^{-}, v_{\mathrm{e}}\right){ }^{20} \mathrm{~F}\left(2_{1}{ }^{+}\right)$ obtained by shell-model calculations with the USDB at $\log _{10} \mathrm{~T}$ $=8.6$. Results obtained by the prescription assuming an allowed GT transition are also shown.

Heating of the $\mathrm{O}-\mathrm{Ne}-\mathrm{Mg}$ core due to $\gamma$ emissions succeeding the double e-capture reactions, ${ }^{20} \mathrm{Ne}\left(\mathrm{e}^{-}, v_{\mathrm{e}}\right)$ ${ }^{20} \mathrm{~F}\left(\mathrm{e}^{-}, \mathrm{v}_{\mathrm{e}}\right){ }^{20} \mathrm{O}$, is important in the final stage of the 
evolution of the core. Study of the evolution of the high density electron-degenerate core with the use of the present shell-model rates is in progress [33].

\section{Summary}

Forbidden transitions in ${ }^{16} \mathrm{O}$ and ${ }^{20} \mathrm{Ne}$ are studied by shell-model calculations with the use of new shellmodel Hamiltonians. Spin-dipole strength in ${ }^{16} \mathrm{O}$ is investigated based on non-energy-weighted and energyweighted sum rules. Total and partial v-induced reactions on ${ }^{16} \mathrm{O}$ in various channels including multiparticle emissions are obtained with the SFO-tls, which can reproduce low-lying spin-dipole states in ${ }^{16} \mathrm{O}$. Total cross sections are found to be close to those of a standard CRPA calculation, while a large branching ratio is noticed for the $\alpha p$ emission channel. This results in an enhancement of production yields of ${ }^{11} \mathrm{~B}$ and ${ }^{11} \mathrm{C}$ in supernova explosion compared with the case without the multi-particle emission channels. Possible measurement of future supernovae at SuperKamiokande by charged-current reactions on ${ }^{16} \mathrm{O}$ is discussed, and dependence of the cross sections on the $v$ mass hierarchies in MSW $v$ oscillations is investigated.

Electron-capture rates for a second-forbidden transition ${ }^{20} \mathrm{Ne}\left(0_{\text {g.s. }}{ }^{+}\right) \rightarrow{ }^{20} \mathrm{~F}\left(2_{1}^{+}\right)$are evaluated by shellmodel calculations with the USDB, and compared with those of the GT prescription, where the transition is treated as an allowed GT transition. Sizable difference is noticed in the e-capture rates in stellar environments, which is caused by the difference in the electron energy dependence of the transition strengths between the two methods.

The authos would like to thank K. Nakazato and M. Sakuda for useful discussion on the $v$ detection at Super-Kamiokande. They also thank S.-C. Leung and S. Zha for useful discussions and studies on the evolution of the O-Ne-Mg core. This work has been supported in part by JSPS KAKENH Grant Nos. JP15K05090 and JP19K03855. K.T. was supported by the JSPS Overseas Research Fellowships

\section{References}

1. T. Suzuki, H. Toki, K. Nomoto, ApJ 817, 163 (2016)

2. H. Toki, T. Suzuki, K. Nomoto, S. Jones, R. Hirschi, Phys. Rev. C 88, 015806 (2013)

3. S. Jones, R. Hirschi, K. Nomoto et al., ApJ 772, 150 (2013)

4. M. Honma, T. Otsuka, T. Mizusaki, M. HjorthJensen, B.A. Brown, JPhCS 20, 7 (2005)

5. K. Mori, M. Famiano, T. Kajino, T. Suzuki et al., ApJ 833, 179 (2016)

6. T. Suzuki, S. Chiba, T. Yoshida, T. Kajino, T. Otsuka, Phys. Rev. C 74, 034307 (2006)

7. T. Yoshida, T. Suzuki, S. Chiba, T. Kajino et al., ApJ 686, 448 (2008)

8. T. Suzuki, A.B. Balantekin, T. Kajino, Phys. Rev. C 86, 015502 (2012)
9. T. Suzuki, M. Honma, Phys. Rev. C 87, 014607 (2013)

10. T. Suzuki, M. Honma, K. Higashiyama, T. Yoshida, T. Kajino, T. Otsuka, H. Umeda, K. Nomoto, Phys.Rev. C 79, 061603 (2009)

11. T. Suzuki, T. Kajino, J. Phys. G 40, 083101 (2013)

12. P. Möller, J.R. Nix, K.-L. Kratz, ADNDT 66, 131 (1997) ; P. Möller, B. Pfeiffer, K.-L. Kratz, Phys. Rev. C 67, 055802 (2003)

13. T. Suzuki, T. Yoshida, T. Kajino, T. Otsuka, Phys. Rev. C 85, 015802 (2012)

14. T. Suzuki, S. Shibagaki, T. Yoshida, T. Kajino, T. Otsuka, ApJ 859, 133 (2018)

15. T. Suzuki, R. Fujimoto, T. Otsuka, Phys. Rev. C 67 044302 (2003)

16. S. Cohen, D. Kurath, Nucl. Phys. 73, 1 (1965)

17. T. Suzuki, T. Otsuka, Phys. Rev. C 78, 061302 (2008)

18. T. Suzuki, S. Chiba, T. Yoshida, K. Takahashi, H. Umeda, Phys. Rev. C 98, 034613 (2018)

19. T. Suzuki, H. Sagawa, Nucl. Phys. A 637, 547 (1998)

20. T. Suzuki, D. F. Measday, and J. P. Roalsvig, Phys. Rev. C 35, 2212 (1987)

21. K. Langanke, P. Vogel, and E. Kolbe, Phys. Rev. Lett. 76, 2629 (1996); E. Kolbe, K. Langanke, and P. Vogel, Phys. Rev. D 66, 013007 (2002)

22. L. Wolfenstein, Phys. Rev. D 17, 2369 (1978) ; D 20, 2634 (1979) ; S.P. Mikheyev, A.Y.Smirnov, Sov. J. Nucl. Phys. 42913 (1985)

23. K. Nakazato, T. Suzuki, M. Sakuda, PTEP 2018, 123E02 (2018)

24. G. Martinez-Pinedo, Y.H. Lam, K. Langanke, R. G. Zegers, C. Sullivan, Phys. Rev. C 89, 045806 (2014)

25. National Nuclear Data Center on-line retrieval system, http://www.nndc.bnl.gov

26. O.S. Kirsebom, et al., arXiv:1805.08149 (2018)

27. J.S. O’Connell, T.W. Donnelly, J.D. Walecka, Phys. Rev. C 6, 719 (1972); J.D. Walecka, in Muon Physics, edited by V.M. Hughes, C.S. Wu (Academic Press, New York, 1975), pp. 113-218; N. Parr, G. Colo, E. Khan, D. Vretenar, Phys. Rev. C 80, 055801 (2009)

28. T. Suzuki, S. Zha. S.-C. Leung, K. Nomoto, ApJ 881, 64 (2019)

29. N. Itoh, N. Tomizawa, M. Tamamura, S. Wanajo, ApJ 579, 380 (2002)

30. S. Ichimaru, Rev.Mod. Phys. 65, 255 (1993)

31. W.L. Slattery, G.D. Doolen, H.E. DeWitt, Phys. Rev. A 26, 2255 (1982)

32. A. Juodagalvis, K. Langanke, W.R. Hix et al, Nucl. Phys. A 848, 454 (2010)

33. S. Zha, S.-C. Leung, T. Suzuki, K. Nomoto, ApJ submitted, arXiv:1907.04184 (2019) 